DOI: https://doi.org/10.46296/yc.v5i9edespjul.0106

\title{
LA GESTIÓN DE OPERACIONES CON ENFOQUE DE GESTIÓN DE RIESGOS EN EL TURISMO
}

\section{MANAGEMENT OF OPERATIONS WITH A RISK MANAGEMENT APPROACH IN TOURISM}

\author{
Verduga-Pino Alexandra María ${ }^{1}$; Pérez-Figueredo Alexis Santiago ${ }^{2}$ \\ ${ }^{1}$ Doctora en Ciencias Económicas. Profesora Titular Universidad Técnica De Manabí, \\ UTM. Portoviejo, Ecuador. Correo: alexandra.verduga@utm.edu.ec. ORCID ID: \\ https://orcid.org/0000-0003-4864-0499
}

${ }^{2}$ Doctor en Ciencias Económicas. Profesor Titular Universidad Oriente. Santiago de Cuba, Cuba. Correo: alexis.figueredo@uo.edu.cu. ORCID ID: https://orcid.org/00000003-3797-0513

\begin{abstract}
Resumen
Objetivo analizar la gestión de riesgos en el contexto de la gestión de operaciones en el sector del turismo favoreciendo el proceso de toma de decisiones. Métodos: La revisión bibliográfica, documental e informes técnicos para la construcción del marco teórico y metodológico actualizando el estado del arte y pertinencia del tema. Los métodos comparativos, el descriptivosexplicativo favorecieron establecer comparaciones y definir los criterios asumidos por el autor. El análisis causal para fundamentar las relaciones entre las variables de la investigación. Resultados. Se propone un procedimiento que desde el enfoque de la gestión de riesgo permite la evaluación de vulnerabilidades que pueden influir en la toma de decisiones afectando la gestión de operaciones en el sector del turismo. A modo de conclusiones, la gestión del riesgo debe verse como un eje transversal en la gestión de cualquier empresa su aplicación en la evaluación de las vulnerabilidades que pudieran afectar la toma de decisiones en la gestión de operaciones en el sector del turismo constituye un enfoque novedoso y pertinente y se convierte en una herramienta que pudiera favorecer el desarrollo y el logro de los objetivos y metas de la empresa turística.
\end{abstract}

Palabras claves: Gestión de riesgos, gestión de operaciones, turismo, vulnerabilidad, toma de decisiones.

\begin{abstract}
Objective: to analyze risk management in the context of the management of operations in the tourism sector, favoring the decision-making process. Methods: The bibliographic and documentary review and technical reports for the construction of the theoretical and methodological framework updating the state of the art and relevance of the subject. The comparative, descriptive-explanatory methods favored establishing comparisons and defining the criteria assumed by the author. The causal analysis to support the relationships between the variables of the investigation. Results: A procedure is proposed that from the risk management approach allows the assessment of vulnerabilities that may influence decision-making, affecting the management of operations in the tourism sector. By way of conclusions, risk management should be seen as a transversal axis in the management of any company, its application in the evaluation of vulnerabilities that could affect decision-making in the management of operations in the tourism sector constitutes a novel approach and relevant and becomes a tool that could favor the development and achievement of the objectives and goals of the tourism company.
\end{abstract}

Keywords: Risk management, operations management, tourism, vulnerability, decision making.

Información del manuscrito:

Fecha de recepción: 17 de mayo de 2021.

Fecha de aceptación: 08 de julio de 2021.

Fecha de publicación: 09 de julio de 2021. 


\section{Introducción}

Las primeras década del siglo XXI se han caracterizado por la agudización de las manifestaciones de diferentes problemas que afectan al mundo y que los procesos de la globalización han favorecido el reconocimiento de sus impactos a una escala micro y local, como los asociados a los cambios tecnológicos, la revolución en las comunicaciones, las crisis económica y ambiental, al cambio climático, los derivados de los conflictos sociales que conmueven diferentes regiones del planeta, y desde finales del 2019 los asociados a la Covid 19, todos en sus conjunto han generado variadas y cada vez más crecientes incertidumbres en todos los sectores de la economía mundial y en especial conformando escenarios muy complejos para el desarrollo del turismo.

Lo anterior permite fundamentar la importancia que han adquirido los estudios de los riesgos como una necesidad imperiosa en la toma de decisiones en los procesos de desarrollo económico y social, de inversiones, de proyectos, en la gestión empresarial, entre otros procesos, favoreciendo desde estas posiciones una mayor comprensión para la búsqueda de posibles soluciones a problemas, que por sus impactos, puedan constituirse en riesgos, generando estados de contingencias o desastres influyendo negativamente en la gestión del desarrollo de un sector, un territorio o una empresa.

Al analizar los aspectos relacionados con la construcción del riesgo por su relevancia, no son los riesgos naturales o tecnológicos y sus consecuencias los únicos que se manifiestan como preponderantes para su estudio.

A nivel microeconómico es posible identificar eventos, que si bien no ocupan la atención mediática de las grandes catástrofes, por el nivel que alcanzan sus manifestaciones deben constituir una prioridad su gestión, se estima que por cada evento extremo que se tratan de manera intensiva a nivel macro, ocurren en las diferentes dimensiones de la sociedad alrededor de 155 eventos a nivel local, de los cuáles entre un 30 y $40 \%$ de ellos tienden a convertirse en situaciones de emergencias o desastres, si no son correctamente gestionados, según presentaciones de la Oficina Regional ISDR en el 
11no Congreso sobre Gestión de Desastres celebrado en la Habana, Cuba, en junio del 2014.

En la práctica cotidiana el riesgo se entiende como sinónimo de inseguridad, describe una situación con un resultado incierto. A partir de lo cual se puede aceptar que, al menos implícitamente, el riesgo está asociado a un grado de incertidumbre que condiciona el saber y no saber, al modo de cómo y para qué actuar, pudiendo ser reconocida esta falta de información que genera un determinado grado de incertidumbre, como el primer peligro que mal gestionado puede derivar en uno de los riesgos primarios a que se enfrenta el hombre en su progreso social. Si la confianza y seguridad son algo deseable, el riesgo es el antónimo, que generalmente entraña impactos negativos o inconvenientes que dificultan el logro satisfactorio de una acción o consecución de un objetivo.

La exposición, susceptibilidad y predisposición de los territorios, de la organización o la empresa, ante un peligro, junto a la disponibilidad y suficiencia de recursos que configuran a la capacidad de respuesta y que potencian la adecuada gestión de las diferentes vulnerabilidades ante los mismos, condicionan el posible nivel de las pérdidas y daños que puede causar la materialización del riesgo. (Pérez, 2014)

Su pertinente estudio favorece el reconocimiento de posibles impactos derivados de la aplicación de las diferentes políticas de desarrollo, globales, territoriales empresariales, que pudieran conllevar a desestabilización económica y social, reproduciendo escenarios de potenciales riesgos sistémicos con consecuencias a largo, mediano y corto plazo, pudiendo generar en su expresión una inadecuada gestión de los recursos, el incumplimiento de los objetivos de desarrollo de la empresa, influyendo en su imagen, la competencia y calidad de sus producciones entre otros impactos.

En el sector de turismo maximizar la satisfacción del cliente significa garantizar la calidad de los servicios y a la vez del producto turístico que se oferta, esto implica el análisis previo de preferencias y de expectativas del turista que deben ser tomada en cuenta de manera 
continuada en la preparación y oferta del producto turísticos.

Esto obliga a la revisión de cuáles son las disponibilidades de recursos, su calidad y vigencia (en el caso de los alimenticios y gastronómicos, por ejemplo) el control y calidad en la ejecución de mantenimientos, revisión de la disponibilidad de almacenes, entre otras acciones que constituyen elementos fundamentales en la gestión de operaciones y logística del turismo.

De la anterior situación descripta emerge el objetivo de esta investigación encaminado a: analizar la gestión de riesgos en el contexto de la gestión de operaciones en el sector del turismo favoreciendo el proceso de toma de decisiones.
La metodología empleada se basó en la revisión bibliográfica, documental y de informes técnicos que permitió construir el marco de referencia teórico y metodológico para este estudio actualizando el estado del arte y pertinencia del tema. El uso de los métodos comparativos, el descriptivosexplicativo favorecieron establecer comparaciones entre diferentes posiciones de autores, así como, definir los criterios asumidos por el autor a lo largo del proceso investigativo.

Para el desarrollo del trabajo se tuvo en cuenta el siguiente esquema metodológico.

Figura 1. Esquema metodológico del trabajo.

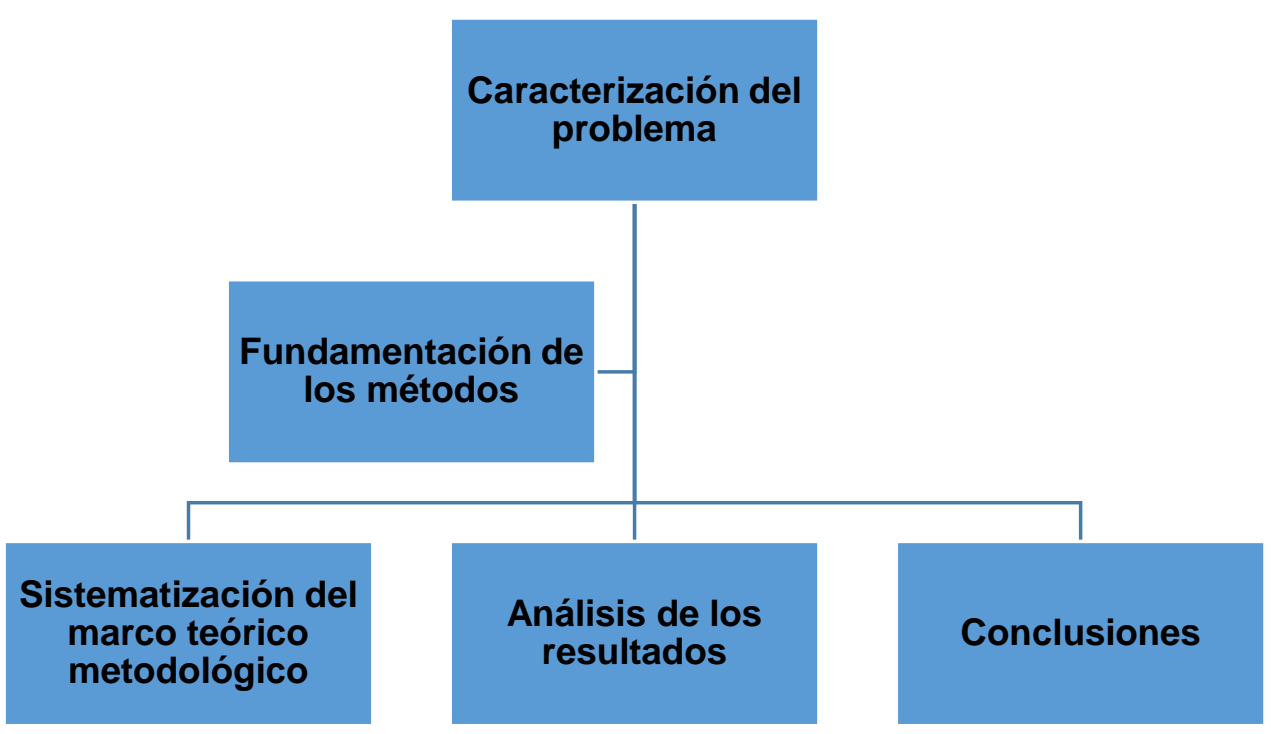




\section{Resultados y discusión}

Resultado de la revisión bibliográfica se contrastan diversas opiniones de autores que han abordado el tema de los riesgos y que posibilitan reflexionar teniendo en cuenta los siguientes enfoques:

Desde las ciencias naturales e ingenieriles: desde esta visión se pone el acento en los procesos naturales y los tecnológicos. Se focaliza en el estudio de los factores desencadenantes, las interrelaciones, la periodicidad, incertidumbre, magnitud y probabilidad de ocurrencia de un evento determinado, lo que es de importancia significativa para la mejora de los sistemas de aviso temprano. Se ocupa de las posibilidades de modelación de las complejas estructuras causa-efectos y finalmente del desarrollo de medidas adecuadas de prevención. En esta concepción es insuficiente la percepción social del riesgo pues, se focaliza siempre el peligro tangible que pueda ser localizable espacialmente. Lo que configura la perspectiva de las ciencias naturales e ingenieriles básicamente como una visión objetivista, para la cual la cuantificación, cálculos de probabilidad y complejas modelaciones representan instrumentos metodológicos básicos. (Coy, 2010).

Para el sector del turismo, es criterio de los autores, esta visión pudiera facilitar las perspectivas en la ejecución y control de los mantenimientos $\mathrm{e}$ inversiones programadas pero la no visibilidad de la percepción social del riesgo limita su observancia en un modelo propio para el turismo.

\section{Desde las ciencias económicas:}

En este caso se trata básicamente de calcular, cuantificar los costos financieros y efectos económicos relevantes de impactos asociados a los eventos generadores del riesgo. En los últimos años se volvió cada vez más importante dentro de la gestión de las empresas la administración de los riesgos y fundamentalmente los riesgos financieros, que centran su atención en la evaluación y cálculos de la magnitud del evento en relación con los daños potenciales y pérdidas que puedan ocasionar, los que se consideran los factores básicos para la estimación del riesgo. En esta posición se consideran los impactos 
económicos y financieros, pero es insuficiente el tratamiento de otros puntos de vistas que limitan por ejemplo la consideración de los recursos humanos y la percepción social del riesgo. Esta perspectiva también está caracterizada por una visión objetivista. (Coy, 2010).

Los autores consideran que para el sector del turismo esta visión aporta elementos contundentes en la gestión económica que influyen en la rentabilidad y eficiencia de la gestión del turismo, pero al igual que los enfoques del riesgo desde las ciencias naturales $e$ ingenieriles limita su desempeño en un modelo propio para el turismo.

Desde las ciencias sociales: en contraposición a las dos perspectivas precedentes, las ciencias sociales no se basan generalmente en la cuantificación o modelación de la magnitud de los daños y pérdidas, sino, más bien en la inclusión social de la gestión de los riesgos focalizando la actuación y de qué forma y en qué medida las personas 0 grupo social, la comunidad, está particularmente expuesto 0 es susceptible a determinado peligro. ¿Cómo influyen las condiciones contextuales y las decisiones individuales en la creación y disponibilidad de la capacidad de respuesta para enfrentarse a ellos? ¿Cuál es la percepción del riesgo de los actores sociales? ¿Cómo han sido diseñadas las estrategias de gestión de riesgos y cuál ha sido la participación de diferentes actores sociales en su concepción? Este tipo de preguntas dominan la perspectiva de las ciencias sociales (sociología, politología, psicología) para la gestión del riesgo. (Coy, 2010).

El resultado del análisis de estos enfoques, permite considerar a los autores que a pesar de la diversidad en los detalles, todas estas posiciones tienen en común un fuerte abordaje cualitativo. Son portadores de un carácter constructivista, por el cual los riesgos y la forma de gestionarlos se interpretan, por ejemplo, como forma de expresión de la cambiante relación hombre-naturaleza, su valoración se ve influenciada por diferentes discursos y distintas formas de acción social que le otorgan determinada ambigüedad y finalmente de distintos intereses y relaciones de poder y dependencia que tienen un papel fundamental en 
la predisposición, susceptibilidad y exposición a los riesgos.

Así mismo es criterios de los autores que en función de la gestión del riesgo los tres planteamientos son básicos para argumentar la necesidad de potenciar la gestión para la reducción de las vulnerabilidades pues constituyen el elemento estratégico de la gestión de los riesgos al ser considerada como el objeto de esta. Por lo que debe ser examinado como un eje transversal que transcurre a lo largo del modelo de gestión turística y que debe ser considerado en el análisis de sus diferentes actividades desde el diseño y la promoción del producto turístico hasta todas aquellas acciones que aseguran su ejecución y calidad.

De manera general los conceptos de riesgo tienen un componente analítico, así como también un componente normativo, que tiene el objetivo social de lograr condiciones que permitan reducir las inseguridades y minimizar la probabilidad de generación de daños y pérdidas siendo aún insuficiente en este análisis la implicación social.
Los riesgos por sus impactos van perdiendo sus fronteras, eso significa que su repercusión tienen efectos más allá del sitio en que se originan, de los límites de regiones y países, se reconocen a nivel global y se anonimizan, es decir que cada vez es más difícil identificar un causante y un responsable. Pero al mismo tiempo determinan que la búsqueda de una solución mantiene su vigencia a nivel local y en los métodos en que se realiza su enfrentamiento. (Pérez, 2014)

Un ejemplo de lo anterior está presente en la expresión del principio de la responsabilidad común pero diferenciada, al analizar en el aprovechamiento de los recursos naturales y los problemas ambientales generados que en su manifestación global configura la crisis ambiental que afecta al planeta.

La forma de hablar sobre riesgos, la manera en que están incorporados en procesos de interacción social, son aspectos básicos de la comunicación y del conocimiento que se tiene y que determinan posiciones éticas respecto a los mismo potenciando las diferentes formas de su gestión y que en su 
conjunto expresan la formación de una cultura de la prevención (Beck, 1994).

Ampliando este planteamiento, es criterio de los autores que la producción social de riqueza, facilitada entre otras por la producción industrial, la globalización y la aplicación de políticas neoliberales, fertiliza la producción de riesgos sistémicos que repercuten en las posibilidades de satisfacción de las necesidades y expectativas, potenciando la construcción de una sociedad de carencias y del descarte, caracterizada por la creación o profundización de vulnerabilidades y acentuando la distribución del riesgo.

Continuando profundizando en esta línea de planteamientos, los autores consideran que la aplicación de las diferentes políticas del desarrollo de los territorios o empresas, pueden profundizar 0 crear nuevas vulnerabilidades cuando no se tienen en cuenta consecuentemente las vulnerabilidades que se tienen ante los riesgos y que el hombre con el apoyo de la ciencia y la técnica puede potenciar el nivel y la capacidad de respuesta así como, la resiliencia existente en el territorio, la comunidad o de que sea portadora la persona pudiendo desarrollar acciones para ser mitigar, reducir sus impactos o adaptarse a los potenciales riesgos derivados de su aplicación.

Por otra parte, entre los impactos de los riesgos pueden considerarse algunos que afectan la salud humana, las condiciones de vida, ocasionar daños y pérdidas en infraestructuras sociales, viviendas con sus lógicas repercusiones sociales o conducir a la manifestación de riesgos derivados, sistémicos que se generan a partir de eventos centrales afectando las dimensiones sociales, económicas, políticas entre otras.

La predisposición, susceptibilidad, capacidad de respuesta, resiliencia, nivel de exposición son elementos que connotan a la vulnerabilidad, estos configuran un escenario interno extrínseco a influencias externas y que son portadores de una carga social para asumir y gestionar al riesgo. De este modo, el riesgo está relacionado básicamente con las decisiones y es en este contexto que se debe analizar a fondo la responsabilidad personal de los actores. 
El conocimiento del riesgo posibilita evaluar de manera proactiva cuáles son las condiciones que tienen el territorio, empresa o grupos de personas de ser afectados por determinado peligro y posibilita actuar de manera estratégica sobre la vulnerabilidad para reducirla 0 desarrollar capacidades adaptativas favoreciendo la gestión del riesgo en la gestión del turismo.

El problema de no contar con estrategias adecuadas que permitan desarrollar la gestión de riesgos en el turismo, circunscribe el alcance que puede tener la acción preventiva induciendo prácticamente que predomine el enfoque reactivo que en la actualidad conducen, generalmente, la confección de los planes de gestión de riesgos y se limite la capacidad del territorio o la entidad turística para evaluar y dar respuesta a una emergencia determinada.

Si bien es posible adoptar decisiones de política con cierto tipo de aproximaciones, el hecho de no evaluar correctamente el riesgo, de no identificar cuáles son sus factores de riesgos conducentes, hacen que la disposición y uso de recursos no sea lo eficaz y dé los resultados tan eficientes como lo que se han querido lograr, limitando el proceso de toma de decisiones desde la perspectiva de la gestión de las entidades o de los territorios.

Para su tratamiento, es necesario crear una visión compartida de la gestión del riesgo en los planes de desarrollo a mediano y largo plazo, garantizando que esta gestión sea asumida como el fin de las actividades desplegadas y no como un componente adicional de estos, por lo que no puede ser considerada solamente como un objetivo del desarrollo local conducente a una solución como meta, sino también como expresión de un pensamiento estratégico.

La evolución hacia el desarrollo de una cultura de la prevención conduce a un cambio en el enfoque dirigido a los elementos que conforman los riesgos y que determinan el impacto de sus consecuencias sobre un sistema, por lo que es viable enfocar la gestión en los factores de riesgos que pudieran potenciar vulnerabilidades y que al ser tratados de forma preventiva sus impactos, puedan ser minimizados 0 se pueden desarrollar acciones adaptativas ante ellos. 
La gestión, dirección o administración de operaciones, como es tratada indistintamente en la literatura, es una actividad que se realiza hacia el interior de las organizaciones para aumentar su capacidad de conseguir los propósitos y objetivos de sus políticas Abarca los cambios en la estructura de la organización y en el sistema de roles y funciones, la elección de personal directivo y asesor, los procesos de capacitación del personal de planta permanente, la mejora continua del funcionamiento de la organización con su actual tecnología y la introducción de innovaciones técnicas y estratégicas acordes con los proyectos de mejora de la calidad, creando organizaciones adaptables, flexibles, controlables, eficientes y competitivas, todo ello dirigido a favorecer la toma de decisiones (Colectivo de autores ,1999).

Existen diversos momentos en la gestión de operaciones:

- Decidir qué, cómo y cuánto producir.

- Con qué recursos producir.
- Diseñar las operaciones necesarias para obtener esas producciones o servicios.

- Con quiénes producir, proyectando su superación y calificación técnica.

- Proyectar y perfeccionar los procesos de la organización e innovar en ellos, para aumentar la calidad, flexibilidad y productividad de los sistemas.

Entre las tareas que debe atender están:

- Planeación estratégica: establecer estrategia de operaciones para apoyar la estrategia general del a organización y desarrollar una estrategia funcional apropiada.

- Planeación de productos: Seleccionar y diseñar los servicios y productos que la organización ofrecerá a sus clientes, patrocinadores 0 receptores.

- Planeación de la capacidad: Determinar cuándo y que tanto las instalaciones, equipo y mano de obra se debe tener disponible.

- Administración de inventarios: Decidir las cantidades de 
materia prima, trabajos en proceso y artículos terminados que conviene almacenar

- Administración del proyecto: Aprender como planear y controlar las actividades del proyecto para cumplir con los requerimientos de desempeño, programa y costo.

- Programación: Determinar cuándo se debe realizar cada actividad o tarea en el proceso de transformación y donde deben estar los insumos.

- Control de calidad: Determinar cómo se deben desarrollar y mantener los estándares de calidad.

Existen condiciones objetivas y subjetivas que limitan una aplicación eficiente de las técnicas o métodos que se agrupan de la gestión de operaciones y que a la vez se pueden convertir en vulnerabilidades que impactan en la toma de decisiones dirigidas a optimizar el logro de los objetivos propuestos por la organización, estas pueden ser:

\section{Objetivas:}

- Recursos.

- Tecnología.

- Entorno.
- Localización.

Subjetivas:

- Calificación del personal.

- Experiencia sobre la técnica.

El desarrollo de la gestión de riesgo desde el contexto de la gestión de operaciones.

De acuerdo con la norma ISO 31000:2018, el riesgo es el "efecto de la incertidumbre sobre los objetivos" y un efecto es una desviación positiva o negativa de lo que se espera. Constituye un proceso dinámico e iterativo que requiere:

1. Formular opciones para el tratamiento de riesgo.

2. Seleccionar la opción más adecuada.

3. Planificar e implementar el tratamiento de riesgos.

4. Evaluar la efectividad de las acciones implementadas.

Estos puntos de vista, unidos a los enfoques de sistema con que son analizados en la actualidad los procesos para la gestión de empresarial, determinan que los 
estudios de los riesgos constituyan más que una necesidad técnica, un imperativo que trasciende a las dimensiones del desarrollo y funciones de la dirección, por lo que deben ser considerados desde los procesos estratégicos de la gestión empresarial a través de los cuáles se establezcan acciones que favorezcan la gestión del riesgo en el logro de este propósito es indiscutible los aportes que puede brindar la gestión de operaciones.

Para lograr incorporar la gestión de riesgos en la gestión de operaciones se requiere identificar y comprender cuáles son los problemas que tiene la organización y evaluar cuales, por sus impactos puede convertirse en peligros y devenir en riesgos para la gestión de operaciones, pero además hacia dentro del proceso en si evaluar los riesgos y para ello es necesario evaluar cómo se comportan las variables de vulnerabilidad y capacidad de respuesta que se tiene para enfrentarlo.

Esto implica identificar entre los problemas que se han generado aquellos que por las posibles pérdidas y daños, frecuencia de manifestación, magnitud y nivel de incertidumbre pueden convertirse en un peligro para la correcta gestión de operaciones y evaluar con precisión, cuál es la capacidad de respuesta para su enfrentamiento o para potenciarla y estimular su desarrollo en el proceso reduciendo las vulnerabilidades asociadas.

Con este procedimiento se amplifica el espectro de la gestión de riesgo más allá del análisis comparativo y reactivo, incorporándolo a la gestión de la empresa teniendo en cuenta la aplicación de las herramientas de la gestión de operaciones

El procedimiento para la incorporación y desarrollo de la gestión de riesgo con este propósito se establece como un conjunto de medidas y acciones que ejecutadas desde el modelo estratégico de la empresa, favorecen la incorporación de la gestión de riesgos desde los diferentes momentos de la gestión de operaciones, asegurando desde la prevención, una respuesta adecuada dirigida a la minimización de las vulnerabilidades o la creación de capacidades de adaptación con el propósito de la reducción del riesgo, así como, beneficiando el desarrollo de sinergias que favorecen el cumplimiento de la legislación 
vigente $\mathrm{y}$ de los requerimientos impuestos a las inversiones y planes de desarrollo que se deben realizar durante el proceso de compatibilización del desarrollo económico - social.

El desarrollo del procedimiento dotará a los tomadores de decisiones en la gestión empresarial de un instrumento de fácil comprensión y aplicación, que ayude a determinar el nivel de riesgos teniendo en cuenta los siguientes puntos de vista: su contextualización, flexibilidad y posibilidades para brindar un tratamiento personalizado al objeto que se estudia.

Su alcance dependerá de la unidad de estudio que se defina. El mismo está estructurado en diferentes fases (Figura 2) las que se desagregan en un conjunto de pasos para dar cumplimiento al objetivo de cada una de ellas y del procedimiento en general.

Figura 2. Procedimiento para incorporar el estudio de los riesgos en riesgo en la gestión empresarial desde la gestión de operaciones.

\begin{tabular}{|l|l|}
\hline - Preparación y diagnóstico. \\
- Caracterización de la unidad de estudio. \\
- Diagnóstico de los principales problemas generados en la gestión empresarial \\
- Caracterización de los principales problemas. \\
Fase 2 \\
- Estudio del riesgo \\
- Identificación y evaluación de los peligros. \\
- Análisis y evaluación de las vulnerabilidades \\
- Análisis y evaluación de las capacidades de respuestas. \\
- Cálculo y estratificación del índice de riesgo. \\
- Propositiva \\
- Elementos estrategicos para incorporar la gestión de riesgo en la gestión \\
empresarial desde la gestión de operaciones. \\
- Plan de acciones. \\
- Divulgación y comunicación de resultados a tomadores decisiones. \\
- Control y retroalimentación
\end{tabular}

Su ejecución toma en consideración el enfoque de proceso, donde cada fase concluida aporta un resultado que constituye el inicio de la siguiente, logrando una interconexión entre sí, garantizando la continuidad del trabajo y estableciendo al mismo tiempo los elementos necesarios de retroalimentación. 
Los resultados de este procedimiento están enfocados a los procesos de toma de decisiones. El alcance de la incorporación de la gestión de riesgo en la gestión empresarial está condicionado por los siguientes factores:

a) La disponibilidad de información básica en las áreas que se estudian;

b) La definición oportuna y cumplimiento de un marco conceptual y legal que integra los distintos procesos;

c) Un abordaje metodológico adecuado con respecto a las condiciones de la unidad de estudio;

d) El nivel de participación efectiva de los actores.

\section{Conclusiones}

Estos argumentos configuran las distintas disquisiciones del riesgo 0 de sus elementos constitutivos comunes a todas las concepciones del riesgos y sus consecuencias inciertas, que pueden ser positivas 0 negativas de acuerdo con la valoración subjetiva, esperadas tras un evento o la inseguridad de su ocurrencia, el conocimiento de su comportamiento son importantes para definir posturas o actitudes desde las cuales se gestiona y que necesariamente dependen de cuál es la percepción que sobre el riesgos poseen los tomadores de decisiones y cómo lo interpretan, lo reconocen y aceptan para precisar acciones de convivencia, mitigación o adaptación ante sus impactos.

Esto se refuerza con la concepción y objetivos de la gestión de operaciones que no se centra en el interés de un evento real en sí, sino en conocer primero, cuáles son las condiciones que favorecen su desarrollo, cómo se genera el riesgo, las vulnerabilidades y percepciones que existe ante el mismo en la empresa y cómo pueden influir en la toma de decisiones.

De esta forma los riesgos no son vistos solamente como un fenómeno de origen externo, sino como, una construcción producida en lo interno, como parte de las acciones cotidianas y como resultado de experiencias, siempre enmarcadas en sus respectivos y específicos contextos empresariales, socioeconómicos y políticos. 
La gestión del riesgo debe verse como un eje transversal en la gestión de cualquier empresa su aplicación en la evaluación de las vulnerabilidades que pudieran afectar la toma de decisiones en la gestión de operaciones en el sector del turismo constituye un enfoque novedoso y pertinente y se convierte en una herramienta que pudiera favorecer el desarrollo y el logro de los objetivos y metas de la empresa turística.

\section{Bibliografía}

Administración de operaciones. (2012). Obtenido de: http://catarina.udlap.mx/u_dl_ a/tales/documentos/mepi/de I_ap/capitulo2.pdf

Administración de operaciones. (2012). Obtenido de: http://rmorales.mayo.uson.mx /admon.pdf

Barrios, G (2008).Monografía. Investigación de operaciones. http://www.eumed.net/librosgratis/2009b/550/La\%20gesti on\%20operativa.htm

Beck, U. (1994). La Sociedad del Riesgo.

Bollin, C. (2015). La gestión de riesgo en el desarrollo territorial. GTZ. Lima. Perú.
CARE_PERU. (2008). Manual para la gestión de riesgos ambientales. Ongs. CARE. Perú

Cáritas del Perú. (2009). Gestión del riesgo de desastres en el desarrollo local. Lima. Perú.

Castellanos, M. (2009). Integración de la dimensión ambiental a la planificación del desarrollo. Revista Cub@: Medio ambiente y desarrollo. Consultada 25/septiembre/2012.pag 38.46

Centro de Estudios para el Desarrollo Económico Facultad de Economía Chile. (2007). Manual técnico estudio de riesgos ambientales. Santiago de Chile

Colectivo de autores, 1999: Libro "Introducción a la investigación de operaciones", tomo III.

Coy, M. (2010) Los estudios del riesgo y de la vulnerabilidad desde la geografía humana. Su relevancia para América latina. Institutfür Geographie, Universität Innsbruck.

Entorno Turístico. (2000). 8 definiciones para entender qué es el turismo. Recuperado de http://www.entornoturistico.co m/8-definicionesparaentender-que-es-el-turismo/ 
Luhmann, N (S/F). Sociología del Riesgo

Muñoz, D. (2009). Administración de operaciones. Enfoque de administración de procesos de negocios. Obtenido de: https://books.google.com.cu/ books/about/Administraci\%C 3\%B3n_de_operaciones_Enf oque_d.html?id=edZx_26yf64 C\&redir_esc=y\&hl=es

Oficina Regional ISDR (2014). 11no Congreso sobre Gestión de Desastres celebrado en la Habana, Cuba.

Pérez F, A.S. (2014). La gestión de riesgos ambientales en las estrategias del DL. Tesis doctorado. Universidad Camagüey. 